\title{
D’une anthropologie des émotions
}

\author{
DAVID LE BRETON ${ }^{1}$ \\ Universidad de Estrasburgo
}

«Tout est fabriqué et tout est naturel chez l'homme, comme on voudra dire, en ce sens qu'il n'est pas un mot, pas une conduite qui ne doive quelque chose à l'être simplement biologique -et qui en même temps ne se dérobe à la simplicité de la vie animale, ne détourne de leur sens les conduites vitales, par une sorte d'échappement et par un génie de l'équivoque qui pourrait servir à définir l'homme» (Maurice Merleau-

Ponty, Phénoménologie de la perception).

\section{Approche NATURALISTE}

L'ANTHROPOLOGIE PROPOSE UNE APPROCHE SYMBOLIQUE du corps et du visage, soulignant la relativité des émotions ou des sentiments selon les situations sociales et culturelles et selon les acteurs en présence. A l'inverse d'autres travaux suivent la postérité de Darwin et actualisent son vocabulaire et ses méthodes en parlant par exemple de programmes génétiques se déployant à leur heure en toute indépendance des données sociales et culturelles. Des mécanismes de déclenchement innés, par imprégnation ou maturation, libèrent le moment venu un programme de comportement qui se manifeste sans que l'éducation exerce d'influence significative. L'expression des émotions relève alors d'une physiologie et non plus d'une symbolique. Fixée au cours de l'évolution à travers des réactions biologiques et l'émission de signaux ayant une utilité particulière à la

1 David Le Breton est professeur de sociologie à l'université Marc Bloch de Strasbourg. Auteur de nombreux ouvrages autour de l'anthropologie du corps. Ouvrages traduits en espagnol : La sociologia del cuerpo (Buenos Aires, Nueva Vision), Antropologia del cuerpo y modernidad (Buenos Aires, Nueva Vision), Las pasiones ordinarias. Antropologia de las emociones (Buenos Aires, Nueva Vision), El silecio (Madrid, Sequitur), Antropologia del dolor (Madrid, Seix Barral). Ou encore de L'adieu au corps (Métailié), La peau et la trace. Sur les blessures de soi (Métailié), La saveur du monde. Une anthropologie des sens (Métailié). 
survie de l'espèce, elle est décrite comme invariante, répondant à des schémas mobilisés par des classes particulières de situation (deuil, perte de statut, gain de pouvoir, etc.) (Kemper, 1978).

L'homme dès lors cesse d'être condition et se voit réduit à l'espèce. Ces approches sont souvent divisées et contradictoires, nous n'en parlerons pas ici, nous l'avons longuement fait ailleurs (Le Breton, 1998). Le naturalisme ne peut guère intégrer le symbolisme social, sinon sous une forme résiduelle. Il est en quête d'un langage naturel des émotions anatomiquement et physiologiquement identifiable. Il repose sur un dualisme opposant les émotions érigées en substance psychologique d'une part (la Joie, la Colère, etc.), et l'individu de l'autre en qui elles transitent provisoirement et s' «expriment» en toute indifférence à ce qu'il est. Ce dernier est écarté, quantité négligeable nuisant à l'émotion théorique qu'il convient de mettre en évidence à travers une série musculaire. Aucune place n'est accordée à l'ambivalence, aux variations personnelles, sociales ou culturelles. Ekman et Friesen, (1984), par exemple, ne voient jamais un visage mais une face morcelée en un écheveau musculaire. Leurs travaux s'appuient uniquement sur une étude méticuleuse de la face. La peau est également éliminée. L'individu pris en compte ressemble tout à fait à une face d'écorché et disposé, sans rancune, à «exprimer » sa joie, son intérêt ou sa surprise, avec les fibres musculaires qui lui restent (Le Breton, 1993). Ecartées également les nuances du regard, les mouvements du corps, leur rythme, les gestes de la main, des épaules, la position du buste, les déplacements, les séquences rituelles qui inscrivent l'émotion dans la durée et la modifient au fil des circonstances, etc. En regardant l'autre nous ne voyons pas une série de contractions musculaires, mais un homme souriant ou amer, avec sur son visage les nuances propres à la singularité de son histoire. Les muscles ne font pas plus le sourire ou la tristesse que le cerveau la pensée, c'est l'homme qui sourit ou qui pense.

Ces perspectives biologiques passent allègrement outre le contrôle relatif opéré sur soi par l'acteur sur ce qu'il veut laisser percevoir de ses sentiments en jouant sur sa manière de se présenter à son public sur la scène sociale. Elles ignorent superbement le théâtre où le comédien suggère par des signes culturels, des émotions qu'il ne ressent pas ou les tables de poker autour desquelles chaque joueur contrôle ses affects et élabore une stratégie de mimiques propres à protéger son jeu et à garantir la meilleure chance. Détachées de la vie réelle ces approches excluent l'ambivalence, le jeu, les variations individuelles (timidité, pudeur, discrétion, contrôle de soi, dissimulation, etc.), les nuances données par les plis du visage, c'est-à-dire la peau nue, texture où se lisent les sentiments qu'un acteur éprouve ou simule en donnant le change. Elles élaguent également les différences sociales et culturelles qui deviennent d'autant plus sensibles quand l'émotion est en situation sollicitant non plus seulement la face, mais 
l'homme en son entier, par tous les mouvements de son corps, sa parole, ses déplacements dans l'espace, etc.

\section{L'ÉMOTION N’EST PAS UNE SUBSTANCE}

Les tenants d'une approche strictement biologique des émotions identifient la haine, l'amour, la jalousie, la joie, la peur, la douleur, etc., comme des objets mentaux repérables comme on chercherait les mille façons de nommer l'eau ou le chien. Manière de les naturaliser sous le prisme d'un vocabulaire dissolvant toute différence, occultant ainsi d'emblée la mosaïque affective des sociétés humaines dans l'espace et le temps. L'émotion n'est pas une substance, un état figé et immuable à retrouver sous une même forme et les mêmes circonstances dans l'unité de l'espèce humaine, mais une tonalité affective qui fait tâche d'huile sur l'ensemble du comportement, et ne cesse de se modifier à tout instant chaque fois que le rapport au monde se transforme, que les interlocuteurs changent. L'émotion n'est pas un objet possédé, ou qui possède, au sens de la transe de possession. Dans l'expérience affective courante l'émotion ou le sentiment ne sont jamais d'une seule teinte, ils sont souvent mêlés, oscillant d'un ton à un autre, marqués d'ambivalence. On peut rire d'une situation ou d'un trait d'humour sans se départir tout à fait de son angoisse à propos de l'attente d'un bilan de santé; se sentir meurtri, et coupable en même temps, après avoir perdu un proche; avoir honte d'une situation en se disant qu'il est temps de repousser une éducation trop pudibonde, etc. L'émotion n'a pas la clarté d'une eau de source, elle est souvent un mélange insaisissable dont l'intensité ne cesse de varier et de se traduire plus ou moins fidèlement dans l'attitude de l'individu.

En faisant de l'émotion une substance biologique les naturalistes travaillent sur un artefact, ils font de notions de sens commun (la joie, la tristesse, etc) des réalités matérielles à retrouver dans les mécanismes neurologiques ou hormonaux. Il n'y a pas un homme qui «exprime» la « joie », mais un homme joyeux, avec un style propre, ses ambivalences, sa singularité. Non pas un homme qui «exprime»sa « détresse » mais un homme en deuil ou meurtri par les événements. Les approches biologiques buttent sur le dualisme (l'homme d'une part, l'émotion de l'autre, comme un état indépendant), l'ambiguïté de la notion d'expression (qui? exprime quoi?), et l'outrance de leurs schèmes d'analyse des visages censés « exprimer » l'émotion. Au double sens du terme, elle est naturalisée. Et l'on recherche les mimiques faciales qui lui correspondent, comme si elle était une quantité finie et sans équivoque, détachable de l'acteur social. Ces travaux distinguent également de manière arbitraire le visage du reste du corps, ils négligent la multitude des signes qui concourent au sentiment éprouvé. Comme si la joie, par exemple, n'était pas accompagnée de regard, de parole, 
d'intonation particulière, et n'impliquait aucun mouvement des mains, des bras, du buste, etc.; mais produisait un simple jeu d'unités musculaires agitant la face. Leur joie parait singulièrement imprégnée d'autisme, ou, au minimum, d'une singulière abstraction. A vouloir les fixer en un schéma simple, en une sorte de portrait robot qui épure à l'extrême toutes les objections possibles, les émotions ne se retrouvent plus nulle part, abstraites, dépouillées du visage qui les dessine, elles ont la dérision d'un croquis voulant s'imposer au paysage.

\section{ANTHROPOLOGIE DES ÉMOTIONS}

L'homme est relié au monde par un permanent tissu d'émotions et de sentiments. Il est à tout instant affecté, touché par les événements. L'affectivité filtre la tonalité du rapport au monde, elle entraîne des modifications viscérales et musculaires. Elle incarne pour le sens commun un refuge de l'individualité, un jardin secret où s'affirmerait une intériorité née d'une spontanéité sans défaut. Pourtant si elle s'offre sous les couleurs de la sincérité et de la particularité individuelle, l'affectivité est toujours l'émanation d'un milieu humain donné et d'un univers social de sens et de valeurs. Si son infinie diversité appartient bien entendu au patrimoine de l'espèce, son actualisation dans un ressenti et une économie subtile de mimiques, de gestes, de postures, une succession de séquences, ne se conçoit pas hors de l'apprentissage, hors du façonnement de la sensibilité que suscite le rapport aux autres au sein d'une culture dans un contexte particulier. L'émotion n'a pas de réalité en soi, elle ne puise pas dans une physiologie indifférente aux circonstances culturelles ou sociales, ce n'est pas la nature de l'homme qui parle en elle, mais ses conditions sociales d'existence qui se traduisent alors par des modifications physiologiques et psychologiques. Elle s'inscrit plutôt à la première personne au sein d'un tissu de significations et d'attitudes qui imprègne simultanément les manières de la dire et de la mettre physiquement en jeu. Elle est donc une émanation sociale rattachée à des circonstances morales, et à la sensibilité particulière de l'individu, elle n'est pas spontanée, mais rituellement organisée, reconnue en soi et signifiée aux autres, elle mobilise un vocabulaire, des discours. Elle relève de la communication sociale. L'individu ajoute sa note particulière et brode sur un motif collectif susceptible d'être reconnu par ses pairs, selon son histoire personnelle, sa psychologie, son statut social, son sexe, son âge, etc. L'affectivité est l'incidence d'une valeur personnelle confrontée à la réalité du monde.

L'éloignement dans l'espace, à travers les données ethnologiques, ou dans le temps, à travers l'histoire des mentalités, souligne les formes changeantes des ressentis et des mises en jeu de l'émotion selon les groupes sociaux et les circonstances. Le détour anthropologique force à se percevoir soi sous l'angle 
de la relativité sociale et culturelle même pour des valeurs qui paraissent intimes et essentielles. Il rappelle le caractère socialement construit des états affectifs même les plus brûlants et de leurs manifestations sur un fond biologique qui n'est jamais une fin mais toujours la matière première sur laquelle brode inlassablement les sociétés (Le Breton, 2004).

\section{L'ÉMOTION NAÎT DE L'ÉVALUATION D'UN ÉVÉNEMENT}

La vie affective s'impose en dehors de toute intention, elle ne se commande guère et parfois elle va à l'encontre de la volonté, mais elle répond toujours à une activité de connaissance liée à une interprétation par l'individu de la situation où il est plongé. Elle est une pensée en mouvement que n'épuise pas le cogito. Des processus inconscients entrent également dans son émergence. Elle autorise parfois un contrôle, du moins un jeu orcés avec son orcésió pour un ajustement plus favorable aux circonstances. Les émotions ne sont pas des turbulences morales percutant des conduites raisonnables, elles suivent des logiques personnelles et sociales, elles ont orc raison. Un homme qui pense est un homme affecté, renouant le fil de sa mémoire, imprégné d'un certain regard sur le monde et sur les autres. Des mouvements affectifs qui paraissent en orcés avec les manières habituelles d'un sujet, ou qui le poussent à agir sur un mode qui lui est nuisible renvoient pour le psychanalyste à des logiques de l'inconscient enracinées à des types de relations noués dans l'enfance et dont la signification peut orc retrouvée au orcé de l'anamnèse. Piaget a mis en évidence qu'il n'y a pas de processus cognitif sans mise en jeu affective et inversement (Piaget, 1989, 75q).

L'individu interprète les situations à travers son système de connaissance et de valeurs. L'affectivité déployée en est la conséquence. Aristote est sans doute le premier à souligner la part active de l'individu dans les émotions qui le traversent. " On doit, en ce qui concerne chaque passion, distinguer orcé points de vue, écrit-il. Ainsi, par exemple, au sujet de la colère, voir dans quel état d'esprit sont les gens en colère, contre quelles personnes ils le sont d'habitude, et pour quel motif » (Aristote, 1991, 183). La signification conférée à l'événement fonde l'émotion ressentie, c'est elle que les propositions orcésións échouent à appréhender du orc des limites de orc cadre de pensée au risque d'élaguer la spécificité humaine qui tient justement dans la dimension symbolique. Dans la terreur qui se saisit d'une foule, dans la haine raciste ou dans les manifestations de la fureur individuelle ou collective, nul triomphe de l' " irrationalité » ou de la « nature », mais la mise en jeu d'un raisonnement, d'une logique mentale, d'une ambiance sociale. Les émotions, écrit Averill (1980, 67), « résultent autant de processus cognitifs complexes que la orcési, l'art ou la science ». On n'est pas 
ému par le déclenchement inopiné d'un processus biologique, mais orc à une implication particulière dans une situation donnée qui mobilise alors un état physiologique reconnaissable. Deux individus entrent en conflit pour une raison quelconque : orc colère flambe ou diminue selon leurs réactions mutuelles, elle disparaît même tout à orc si l'un d'eux reconnaît soudain ses torts. Mais l'un aurait pu aussi rester de marbre orc à la provocation de l'autre connaissant sa orcésión à chercher querelle pour des broutilles. Un homme est effrayé par un bruit suspect dans sa maison; il avance avec crainte, il se rassure en voyant une fenêtre ouverte agitée par le vent. Mais la peur rebondit s'il se souvient de l'avoir fermée auparavant et découvre la poignée orcé. D'un raisonnement à l'autre l'émotion change radicalement de forme. Une interprétation erronée peut induire une vive angoisse créée de toutes pièces, elle n'en est pas moins agissante. On peut se faire peur, ou même se faire mourir en intériorisant la conviction culturelle d'être par exemple victime d'une entreprise sorcellaire.

\section{L'EXPRESSION SOCIALE DES ÉMOTIONS}

A l'intérieur d'une même communauté sociale les manifestations corporelles et affectives d'un acteur sont virtuellement signifiantes aux yeux de ses partenaires, elles se renvoient les unes aux autres à travers un jeu de miroir infini. Son expérience contient en germe celle des membres de sa société. Pour qu'une émotion soit ressentie, perçue, et exprimée par l'individu, elle doit appartenir sous une forme ou sous une autre au répertoire culturel de son groupe. Un savoir affectif diffus circule au sein des relations sociales et enseigne aux acteurs, selon leur sensibilité personnelle, les impressions et les attitudes qui s'imposent à travers les différentes circonstances de leur existence singulière. Les émotions sont des modes d'affiliation à une communauté sociale, une manière de se reconnaître et de pouvoir communiquer ensemble sur le fond affectif proche. « Il y a des gens qui n'auraient jamais été amoureux s'ils n'avaient jamais entendu parler de l'amour » dit finement La Rochefoucault.

Mauss montre comment les sociétés induisent une « expression obligatoire des sentiments » qui imprègne l'individu à son insu et le rend conforme aux attentes et à la compréhension de son groupe. Il dégage la rigoureuse progression sociale d'un rite funéraire australien dont l'affectivité est régie par des règles que les acteurs ne cessent de rejouer en se conformant aux usages. La vive douleur exprimée par les cris, les lamentations, les chants, les pleurs n'en est pas moins sincères. Les manifestations du chagrin diffèrent selon la position des acteurs dans le système de parenté, elles ne sont pas univoques, une dose licite de souffrance est de mise selon la proximité avec le défunt, selon que l'endeuillé est un homme ou une femme. La conclusion de Mauss a une valeur 
programmatique, elle ouvre en son temps un vaste domaine d'analyse : « Toutes ces expressions collectives, simultanées, à valeur morale et à force obligatoire des sentiments de l'individu et du groupe, ce sont plus que de simples manifestations, ce sont des signes des expressions comprises, bref, un langage. Ces cris, ce sont comme des phrases et des mots. Il faut dire, mais s'il faut les dire c'est parce que tout le groupe les comprend. On fait donc plus que de manifester ses sentiments, on les manifeste aux autres puisqu'il faut les leur manifester. On se les manifeste à soi en les exprimant aux autres et pour le compte des autres. C'est essentiellement une symbolique » (Mauss, 1968-1969, 88). ${ }^{2}$

L'affectivité des membres d'une même société s'inscrit dans un système ouvert de significations, de valeurs, de ritualités, un vocabulaire, etc. L'émotion puise à l'intérieur de cette trame donnant aux acteurs une grille d'interprétation sur ce qu'ils éprouvent et perçoivent de l'attitude des autres. Bateson (1986) désigne par ethos «le système culturellement organisé des émotions ». Avec Margaret Mead il reprend ce concept dans Balinese Character (1942). Au sein d'un même groupe un répertoire de sentiments et de conduites est approprié à une situation en fonction du statut social, de l'âge, du sexe de ceux qui sont affectivement touchés et de leur public. Une culture affective est socialement à l'oeuvre. Chacun impose sa coloration personnelle au rôle qu'il joue avec sincérité ou distance, mais un canevas demeure qui rend les attitudes reconnaissables.

\section{Cultures afFectives}

Les différences de cultures affectives se marquent par l'existence d'émotions ou de sentiments qui ne sont pas aisément traduisibles en d'autres langues sans de grossières erreurs d'interprétation. La fidélité aux significations affectives locales implique de conserver le terme vernaculaire ou de recourir à des périphrases afin de les décrire avec nuance et précision. L'écueil de traduction dénote des différences de ressenti et d'expression d'une société et d'une époque à l'autre. Le détour anthropologique montre la relativité culturelle des ethos. En outre, chaque état affectif s'insère dans un ensemble de significations et de valeurs dont il dépend, et dont on ne le détache pas sans le perdre. Une culture affective forme un tissu serré où chaque émotion est mise en perspective à l'intérieur d'un ensemble. Parler des émotions dans l'absolu, comme par exemple de la colère,

2 Durkheim a lui-même esquissé cette analyse: «Le deuil n'est pas un mouvement de la sensibilité privée, froissée par une perte cruelle; C'est un devoir imposé par le groupe. On se lamente, non pas simplement parce qu'on est triste, mais parce qu'on est tenu de se lamenter. C'est une attitude rituelle qu'on est obligé d'adopter par respect pour l'usage, mais qui est dans une large mesure indépendante de l'état affectif des individus» (Durkheim, 1968, 568). 
de l'amour, de la honte, etc., revient à commettre une forme plus ou moins sensible d'ethnocentrisme en postulant implicitement une signification commune à différentes cultures. Les motifs de « honte », par exemple, sont variables selon les groupes sociaux, celle-ci se traduit sous des formes bien différentes qui n'ont souvent aucun rapport avec ce que ressent un individu « honteux » dans nos propres sociétés (Herzfeld, 1980). Il conviendrait de mettre des guillemets à chaque usage d'un terme émotionnel pour traduire le fait qu'il ne s'entend réellement qu'à l'intérieur d'un ethos propre et demeure donc pour l'anthropologue une question posée. Il s'agit d'éviter la confusion entre les mots et les choses et de naturaliser ainsi les émotions en les transportant sans précaution d'une culture à une autre à travers un système de traduction aveugle aux conditions sociales d'existence qui enveloppent l'affectivité. On ne saurait saisir le mouvement complexe de l'émotion sans le mettre en lien étroit, dans une situation précise, avec la manière dont une culture affective propre à un groupe se mêle étroitement à la trame sociale (Myers, 1989; Papataxiarchis, 1994; Vale de Almeida, 1994).

Les ethnopsychologies révèlent bien des particularités sociales et culturelles qui relativisent le répertoire affectif de nos sociétés sur lequel se fondent les naturalistes pour étayer leur conviction d'une universalité de l'émotion. J. Leff $(1977,322)$ fait état de travaux sur la dépression qui trouvent aisément des équivalents sémantiques dans la famille indo-européenne mais échouent face au chinois, au yoruba, et d'autres langues de sociétés non-occidentales. Le recours à des périphrases s'avère nécessaire afin de forger des catégories plus ou moins proches mais loin de la forme et de la durée d'une telle affection pour nos sociétés. « Dans nombre de langues africaines, note-t-il encore, un seul terme signifie le fait d'être triste et d'être en colère » $(1973,301)$. Le concept amaé, considéré par Doi comme une clé pour comprendre la mentalité japonaise, est sans traduction dans d'autres langues et renvoie à une culture affective propre, même s'il se retrouve ici ou là dans le ressenti individuel ailleurs qu'au Japon (Doi, 1988). Les Japonais eux mêmes s'étonnent de l'absence d'un terme proche dans le lexique des langues occidentales où un tel sentiment est restitué par des explications ou des artifices de langage. La définition de Doi suggère les périphrases suivantes: «dépendre de l'amour d'un autre », « se réchauffer » ou « se livrer à la douceur d'un autre ». Amaé souligne une agréable dépendance, la recherche d'une gratification ou l'abandon passif à l'affection d'une autre personne. La conduite de l'enfant à l'égard de sa mère donne l'archétype d'un sentiment qui se poursuit ensuite sur un autre registre. Amae dérive d'ailleurs du verbe amaeru qui signale la dépendance, l'attente d'un traitement favorable. La racine est commune avec amai qui signifie « sucré ». Amae se retrouve dans les relations entre le mari et l'épouse, le maitre et le disciple, etc. Sur le fond de relations dissymétriques fortement présentes dans la culture japonaise, amae introduit une chaleur réconfortante, une douce intimité qui valorise et rend moins tranchante une dépendance personnelle. Amanzuru 
désigne le fait de se contenter, de se soumettre à une situation, de s'en faire une raison, etc. La tonalité affective de prédilection dans les relations inégales réside dans amae, mais si les circonstances rendent impossibles ce sentiment, alors on se contente d'amanzuru. « Les Japonais, écrit encore Doi, pensent que l'usage des mots peut refroidir l'atmosphère alors que les Américains au contraire se sentent encouragés et rassurés par une telle communication. Cela est lié à la psychologie de l'amaé car au Japon ceux qui sont proches l'un de l'autre - ou plutôt ceux qui ont le privilège de se fondre ensemble - n'ont pas besoin de mots pour dire leurs sentiments. Il faut ne pas se sentir en prise sur l'autre (manquer à l'amaé) pour éprouver ainsi la nécessité de verbaliser».

Chez les Kainkang, une société indienne du Brésil, to nu pourrait de prime abord être assimilé à « colère » pour un observateur occidental détaché et indifférent au contexte social. «To indique une direction et $n u$ exprime la colère... et signifie aussi « dangereux ». Une phrase avec l'expression to $n u$ signifie (il est) dangereux. Ainsi, to $n u$ inclut une dimension de danger immédiat, et $n u$ quelque chose d'une colère indirecte. Ainsi la phrase « je suis en colère contre vous » signifie en fait « je suis dangereux pour vous »... Des conspirateurs ne diraient pas « Tuons les », mais plutôt « Soyons en colère contre eux ». Quand Thuli demande à son beau-père de se mettre en colère, il lui demande de commettre un meurtre » (Henry, 1936, 255). Mieux vaut éviter de dire sa colère à un Kainkang car loin du repentir sa réaction serait une manifestation de rage. Même s'il sait que son interlocuteur n'a guère l'intention de lui nuire, une aura dangereuse gravite autour de la colère ( $\mathrm{p} 256)$.

A Samoa musu traduit le refus non motivé de faire quelque chose. Une femme repousse son amant, un bébé ne veut pas se coucher, un chef ne consent pas à prêter sa coupe à kava, etc. Interrogé sur son opposition l'individu avoue son impuissance : «Je me le demande », «Je ne sais pas, c'est tout». Cette attitude est admise, elle se justifie et provoque même « une sorte de respect superstitieux » (Mead, 1963, 381). A Bali, M. Mead repère une association entre la peur et le sommeil qui illustre là encore la particularité de la culture affective. Quand des Balinais sont effrayés ils vont dormir. Cette conduite est nommée d'ailleurs expressément takoet poeles (effrayé endormi). Un jour M. Mead envoit ses aides porter des ustensiles de cuisine en bus dans une habitation où elle doit se rendre. Quand elle arrive plus tard en compagnie de Bateson, elle les découvre assoupis. Ils avaient oublié leur paquet dans le bus et effrayés de la réaction qu'ils craignaient de la part de l'ethnologue, ils s'étaient endormis. La peur est un sentiment contrôlé par le sommeil (Bateson, Mead, 1942, 191)³.

3 De nombreux travaux soulignent la spécificité des cultures affectives (Le Breton, 1998), voir par exemple Rosaldo (1980). Wierzbicka (1988) en recourant à des situations concrètes 


\section{De LA COMÉDIE HUMAINE AU THÉÂTRE}

La culture affective cependant n'est pas une chape de plomb qui pèse sur l'acteur, elle est un mode d'emploi à sa disposition, une suggestion à répondre propre à des circonstances particulières, mais elle ne s'impose pas comme une fatalité mécanique, elle laisse place à des stratégies avantageuses, à des dissimulations. L'acteur est en mesure de « jouer» avec l'expression de ses états affectifs se sentant par exemple trop éloigné de ceux qui seraient socialement adéquats. Un ami le déçoit par ses propos, il demeure en apparence calme et tranquille; il ne ressent aucune douleur à la mort d'un proche, mais s'efforce de prendre un air affligé; il souhaite attirer la compassion sur son état et adopte une attitude larmoyante, il cherche à séduire et endosse les atours de la passion; on lui annonce un échec cuisant pour une demande qui lui tenait à coeur, pour sauver la face il feint l'indifférence et sourit même pour afficher combien l'événement ne l'affecte guère, etc. Les situations sociales abondent en décalages minuscules ou majeurs entre le ressenti du moment et son expression donnée à lire aux autres. Les compétences sociologiques de l'individu incluent bien entendu la maitrise relative de la culture affective selon ce qu'il souhaite montrer de lui-même. S'il déroge aux attentes et s'il y attache de l'importance, il lui est loisible de donner le change par un bricolage personnel sur l'expression de son affectivité. En contrôlant l'image qu'il entend donner de lui-même, il ménage les autres, les manipule, tente ainsi de préserver l'estime de soi, etc. A moins que sa duplicité ne soit connue de longue date ou inopinément révélée. En prodiguant les signes apparents d'une émotion qu'il n'éprouve pas, ou en dissimulant habilement son ressenti, l'individu se construit un personnage, il répond ainsi aux attentes de son public ou satisfait à leur propos l'identité qu'il souhaite produire. L'expression du sentiment est alors une mise en scène qui varie selon les auditoires et selon les enjeux (Hochschild, 1979).

En ce qu'ils semblent afficher les signes de leur bonne foi, le corps et le visage se prêtent à la duplicité. Tout homme dispose de cette faculté de jouer un rôle en se jouant des signes qui annoncent aux autres une signification dont il contrôle soigneusement la portée. Le jeu sur la scène est pensable car la comédie est d'abord dans la vie sociale. Le paradoxe du comédien est le paradoxe de la symbolique corporelle (Le Breton, 1990, 1998), il est le prolongement de

illustre la difficulté de traduction en d'autres langues des termes clés du vocabulaire affectif des Ifaluk tels que les décrit Catherine Lutz (1987). L'anthropologue indien Owen M. Lynch explique en introduction à un ouvrage collectif sur la construction sociale des émotions en Inde que «ces essais posent le problème de la compréhension occidentale des émotions, particulièrement quand celle-ci est universalisée en une pensée et projetée sur l'Autre» (Lynch, 1990, 3). 
la latitude propre à l'homme de témoigner aux autres des seules significations qu'il entend leur donner. La sincérité est malaisément accessible à la pénétration psychologique, la confiance est une croyance sans laquelle le lien social est difficilement pensable. L'apparence est la scène proposée par l'homme du commun à la lecture de ses partenaires. L'art du comédien exploite ce gisement de signes, il en fait un jeu d'écriture qui affiche un état moral de son personnage. L'intelligibilité du spectacle implique la signifiance des mises en jeu du corps du comédien. Simultanément à la parole énoncée, ou en rupture avec elle selon la dramaturgie choisie, le corps se fait lui même récit, il porte le sens de la prestation à part égale avec la parole.

La scène du théâtre est un laboratoire culturel où les passions ordinaires dévoilent leur contingence sociale et se donnent à voir sous la forme d'une partition de signes physiques que le public reconnait d'emblée comme faisant sens (Le Breton, 1998). Le comédien dissipe sa personne dans le personnage, même si les critiques ne se lassent pas de comparer l'un à l'autre, et d'évaluer les différentes prestations qu'ils connaissent autour du même rôle. Mais il ne se confond pas à son personnage, il l'interprète et prodigue à la salle les signes qui établissent l'intelligibilité de son rôle. Il joue, c'est-à-dire qu'il introduit une distance ludique entre les passions sollicitées par son rôle et les siennes, il bricole en artisan sur son corps pour repousser son affectivité de personne singulière et donner leur chance aux émotions de son personnage. Il instruit aux yeux du public une croyance à son rôle grâce au travail d'élaboration fourni à l'aide du metteur en scène. La transmutation n'est possible que parce que les passions ne sont pas érigées en nature, mais sont le fait d'une construction sociale et culturelle, et qu'elles s'expriment dans un jeu de signes que l'homme a toujours la possibilité de déployer, même s'il ne les ressent pas.

Le comédien élabore une émotion à la manière dont un musicien se met au diapason de l'orchestre. Il s'accorde comme un instrumentiste pour entrer dans la musicalité affective de son personnage. «J'observai un jour un grand acteur dans un de ses meilleurs rôles, écrit ainsi Stanislavski. Il attaque un long monologue. Il n'est pas immédiatement tombé sur le sentiment juste; tel un chanteur, il cherche le la. Voici. Non, trop bas; trop haut. Enfin, il a reconnu le bon, a compris, senti, réglé; le voici d'aplomb; il peut maintenant jouir de son art. Il parle librement, simplement, d'un ton plein et inspiré. Il croit à ce qu'il fait » (Stanislavski, 1950, 188). La composition du comédien est un travail sur soi, un cisèlement de l'affectivité, des gestes, des déplacements et de la voix qui doit mener à la rigueur physique et morale du rôle tenu. Le ressenti du théâtre n'est pas celui de la vie réelle. Stanislavski évoque par analogie la douleur de l'individu frappé par un drame et impuissant à communiquer. Avec le temps « on peut enfin parler de ces événements d'une manière cohérente, lentement, intelligiblement, et l'on peut rester maître de soi en racontant l'histoire, tandis 
que ce sont les autres qui pleurent ». Tel est l'objectif que Stanislavski assigne au comédien : "C'est pour cela que notre art exige qu'un acteur expérimente les angoisses de son rôle, qu'il pleure toutes les larmes de son corps chez lui ou pendant les répétitions, de façon à attendre le calme, de façon à se débarrasser de tous les sentiments étrangers à son rôle ou pouvant lui nuire. Il peut alors paraître sur la scène pour communiquer au public les angoisses qu'il a traversées, mais en termes clairs, prenants, profondément ressentis, intelligibles et éloquents. A ce moment le public sera plus affecté que l'acteur, et celui-ci conservera toutes ses forces pour les diriger là où il a le plus besoin d'elles pour reproduire la vie intérieure du personnage qu'il représente » (Stanislavski, 1966, 75).

Le comédien est le « joueur » professionnel d'un clavier d'émotions dont il est un fin observateur. Il est un maître de la duplicité. Cette faculté de se détacher de ses sentiments propres et de donner le change grâce à l'usage approprié de signes, il en fait son métier et son talent. Il se dédouble et s'observe plongé dans la détresse ou la révolte, la joie ou la violence, le rire ou les larmes. Parfois d'une scène à l'autre il est amené à modifier en profondeur la psychologie de son personnage. D'où la formule de Antonin Artaud faisant de lui un « athlète affectif » (Artaud, 1964, 195), un homme capable d'endosser sans transition, et sans lien avec son ressenti propre, les apparences extérieures des émotions ou des sentiments requis par son rôle après en avoir essayé différentes versions. La structure anthropologique du théâtre consiste dans ce privilège de l'homme de se jouer des signes pour les rendre agissant, même s'il n'y croit qu'à demi. Comme dans la vie courante, la sincérité n'est qu'un artifice de mise en scène, un art de se présenter judicieusement au jugement de l'autre en lui laissant voir ce qu'il est tout prêt à accréditer. S'il donne à lire les brûlures de la jalousie, Orson Welles n'est pas Othello. Il satisfait chaque soir aux exigences de son rôle et il y a toujours un moment où le personnage prend enfin congé de la personne. Le comédien joue symboliquement de l'instrument de travail qu'est son corps. Il en fait jaillir les formes imaginaires en puisant dans le fond commun de signes qu'il partage avec son public. Son talent consiste dans le supplément qu'il suscite par sa personnalité propre, son aptitude à emporter l'adhésion de la salle. Il ne s'agit pas de reproduire un texte, mais de l'incarner, de le rendre vivant aux yeux de l'auditoire. Etre un Othello crédible, avec cet ajout subtil dans le jeu qui fait date et rappelle que le comédien est un artiste et non un simple reproducteur. Sa professionnalité est une mesure de son aisance à se mouvoir à l'intérieur des codes d'expression propres à son public.

Le paradoxe du comédien consiste dans cet art de façonner les signes, de faire de son corps une écriture intelligible, afin de déployer à heure fixe une hilarité à gorge déployée pour une réplique entendue mille fois ou les affres de la douleur ou de la jalousie. Il joue indifféremment la joie, la douleur, la mélancolie, simplement en puisant dans un répertoire social et culturel. Il est 
peut-être frappé d'un deuil et rongé par le chagrin, mais quand il entre en scène, il se fond dans les conventions de conduites de son personnage, et il en rend la psychologie crédible en devenant un sociologue attentif à son expression corporelle et orale (Le Breton, 2004). Sur le plateau il déclare sa flamme à une partenaire exécrée, car il s'agit pour lui d'être un orfèvre en l'art de présenter des sentiments qu'il ne ressent pas et se fabrique provisoirement pour les nécessités de son rôle. Il nourrit son jeu des nuances affectives disponibles dans le registre symbolique de son groupe. "C'est la tête du comédien qui porte quelquefois un trouble passager dans ses entrailles, écrit D. Diderot (1967, 133-134); il pleure comme un prêtre incrédule qui prêche la Passion; comme un séducteur aux genoux d'une femme qu'il n'aime pas, mais qu'il veut tromper; comme un gueux dans la rue ou à la porte d'une église, qui vous injurie lorsqu'il désespère de vous toucher; ou comme une courtisane qui ne sent rien, mais qui se pâme entre vos bras ». Diderot lie à sa manière la scène sociale et la scène de théâtre sous la même fiction agissante des signes.

La duplicité est la condition même de l'art du comédien se refaisant professionnellement chaque soir pendant des mois le visage de son personnage sans égard pour ses sentiments propres. La qualité du jeu implique la distance et l'écriture symbolique sur le corps. Diderot à raison de dénoncer la facticité de la sensibilité comme principe de la prestation. Le comédien est un inventeur d'émotions qui n'existent pas à l'état brut, mais qu'il façonne avec son talent personnel en se jouant de signes expressifs socialement reconnaissables. Il développe une connaissance précise des mises en jeu rituelles de la parole et du corps dans les différentes circonstances de la vie sociale. La sociologie du corps est sans secret pour lui. «Il me faut dans cet homme, dit encore Diderot, un spectateur froid et tranquille; j'en exige, par conséquent, de la pénétration et nulle sensibilité, l'art de tout imiter, ou, ce qui revient au même, une égale aptitude à toutes sortes de caractères et de rôles » (p 127-128). De manière saisissante le comédien nous rappelle, à l'encontre des approches naturalistes de l'émotion que celle-ci est conventionnelle et que son expression relève d'un jeu de signes, même dans une brûlante sincérité.

Les sentiments et les émotions ne sont pas des substances transposables d'un individu et d'un groupe à l'autre, ce ne sont pas, ou pas seulement, des processus physiologiques dont le corps détiendrait le secret. Ce sont des relations. Si l'ensemble des hommes de la planète dispose du même appareil phonatoire, ils ne parlent pas la même langue; de même si la structure musculaire et nerveuse est identique, cela ne présage en rien des usages culturels à laquelle elle donne lieu. D'une société humaine à une autre, les hommes ressentent affectivement les événements à travers des répertoires culturels différenciés qui se ressemblent parfois mais ne sont pas identiques. L'émotion est à la fois interprétation, expression, signification, relation, régulation d'un échange, 
elle se modifie selon les publics, le contexte, elle diffère dans son intensité, et même dans ses manifestations, selon la singularité personnelle. Elle se coule dans la symbolique sociale et les ritualités en vigueur. Elle n'est pas une nature descriptible hors de tout contexte et indépendamment de l'acteur.

\section{OuvRAGES CITÉS}

ARISTOTE, Rhétorique, Paris, Livre de poche, 1991.

ARTAUD, A., Le théatre et son double, Paris, Gallimard, 1964.

AVERIL, J.R., "Emotion and anxiety: sociocultural, biological, and psychological determinants", in Rorty A. O. (ed.), Explaining emotions, Berkeley, University of California Press, 1980.

BATESON, G., La cérémonie du Naven, Paris, Biblio-Essais, 1986.

BATESON, G., Mead M., Balinese character: a photographic analysis, New York, New York Academy of Science, 1942.

COSNIER, J., Brossard A. (éd.), La communication non verbale, Delachaux et Niestlé, 1984.

CRAPANZANO, V., "Réflexions sur une anthropologie des émotions", Terrain, $\mathrm{n}^{\circ} 22,1994$.

DIDEROT, D., Le paradoxe du comédien, Paris, Garnier-Flammarion, 1967. DOÏ, T., Le jeu de l'indulgence, Paris, L'Asiathèque, 1988.

DURKHEIM, E., Les formes élémentaires de la vie religieuse, Paris, PUF, 1968.

DUVIGNAUD, J., L'acteur, Paris, Ecriture, 1993.

DUVIGNAUD, J., Le don de rien, Paris, Stock, 1977.

EKMAN, P., Friesen W., "La mesure des mouvements faciaux", in Cosnier J., Brossard A. (1984).

HENRY, J., "The linguistic expression of emotion", American Anthropologist, $\mathrm{n}^{\circ} 38,1936$.

HERZFELD, M., "Honor and shame: problems in the comparative analysis of moral system", Man, n¹9, 1980.

HOCHSCHILD, A. R., "Emotion work, feeeling rules, and social structures", American Journal of Sociology, 85-3, 1979.

KEMPER, T. D., A Social Interactional Theory of Emotions, New York, Wiley, 1978.

LE BRETON, Les passions ordinaires. Anthropologie des émotions, Paris, Petite Bibliothèque Payot, 2004.

LE BRETON, D., Des visages. Essai d'anthropologie, Paris, Métailié, 1992 (2004).

LE BRETON, D., Anthropologie du corps et modernité, Paris, PUF, 1990 (3e édition mise à jour 2005). 
LEFF, J., "The crosscultural study of emotion", Culture, medicine and psychiatry, $\mathrm{n}^{\circ} 4,1977$.

LEFF, J., "Culture and the differenciation of emotion states", British Journal of Psychiatry, $\mathrm{n}^{\circ} 123,1973$.

Lutz, C., Unnatural Emotions, Chicago, The University of Chicago Press, 1988.

LYNCH, O.,

MAUSS, M., "L'expression obligatoire des sentiments", Essais de sociologie, Paris, Minuit, 1968-9.

MEAD, M., Moeurs et sexualité en Océanie, Paris, Plon, 1963.

MYERS, F. R., "Emotions and the self: a theory of personhood and political order among Pintupi Aborigines", Ethos, n'7, 1979.

PAPATAXIARCHIS, E., "Emotions et stratégies d'autonomie en Grèce égéenne", Terrain, n²2, 1994.

STANISLAVSKI, C., La construction du personnage, Paris, Perrin, 1966.

STANISLAVSKI, C., Ma vie dans l'art, Paris, Librairie Théatrale, 1950.

VALE DE ALMEIDA, M., "Emotions rimées. Poétique et politique des émotions dans un village du sud du Portugal", Terrain, n²2, 1994. 
